\title{
EFFECTS OF BLOCK AND DAILY UNDULATING PERIODIZATION ON NEUROMUSCULAR PERFORMANCE IN YOUNG MALE HANDBALL PLAYERS
}

\author{
Rafael Sabido, Jose Luis Hernández-Davó, Javier Botella, Adrián Jiménez-Leiva, \\ and Jaime Fernández-Fernández \\ Sport Research Center, Miguel Hernández University of Elche, Elche (Alicante), Spain
}

Original scientific paper

https://doi.org/10.26582/k.50.1.6

UDC: $796.015 .14: 796.322$

\begin{abstract}
:
The purpose of the present study was to compare block periodization (BP) and daily undulating periodization (DUP) in eliciting changes in strength, jumping performance and throwing velocity in young male handball players. Fifteen players, of the same competitive level, were assigned to one of the periodization groups (DUP=9; $\mathrm{BP}=6$ ). Pre- and post-tests included a one repetition maximum (1 RM) back squat, countermovement jump (CMJ), triple jump with the left and right leg, $20 \mathrm{~m}$ speed test, standing medicine ball throw, $7 \mathrm{~m}$ standing throw, and $9 \mathrm{~m}$ jumping throw. Strength training was performed twice a week throughout an 8-week period. The BP group performed training phases in the following order: hypertrophy, power strength and explosive strength; DUP alternated every week two sessions with a different focus. Total volume and intensity during the 8 -week period were equal in both training groups. Despite both groups significantly improved $1 \mathrm{RM}$ squat $(33.3 \%$, effect size $[\mathrm{ES}]=1.48$ for DUP and $17.4 \%, \mathrm{ES}=1.40$ for $\mathrm{BP})$ and triple jump $(4-6.3 \%, \mathrm{ES}=0.32-$ 0.51 for DUP and $9.7-12.7 \%$, $E S=1.40$ for $\mathrm{BP})$ performance, greater gains $(\mathrm{ES}=0.96)$ were detected in DUP for the $9 \mathrm{~m}$ throwing velocity. No significant changes were found in either group for the $20 \mathrm{~m}$ sprint and CMJ. It can be concluded that both periodization programs elicited significant improvements in maximal strength and jumping performance in young male handball players, while DUP was more effective than BP on throwing velocity.
\end{abstract}

Key words: strength, youth, team sport, training

\section{Introduction}

Team handball is an Olympic sport that requires high intensity bouts of running, sprinting, jumping and throwing (Gorostiaga, Granados, Ibañez, Gonzalez-Badillo, \& Izquierdo, 2006). Additionally, substantial levels of strength are required to optimally hit, block, push and hold during different game situations (Chelly, Hermassi, \& Shephard, 2010; Gorostiaga, Granados, Ibañez, \& Izquierdo, 2005; Marques, Van Den Tillaar, Vescovi, \& Gonzalez-Badillo, 2007). Therefore, a training intervention aimed at increasing strength levels in handball players would improve their on-court handball performance (Gorostiaga, et al., 2006). In particular, strength programs focused on sportspecific actions could improve jumping ability and throwing velocity of players (Hermassi, Chelly, Tabka, Shephard, \& Chamari, 2011). Furthermore, when including strength training throughout the season, maximal strength and jumping performance could be maintained or even enhanced during the competitive phase (Granados, Izquierdo, Ibañez, Ruesta, \& Gorostiaga, 2008).

Including resistance exercises in the training program does not necessarily ensure optimal improvements in explosive force production transferred to sport-specific skills (Faigenbaum, et al., 2009). A vast amount of research supports the notion that the periodized programs provide greater improvements in strength, power, body composition, and performance variables (Fleck, 1999) than the non-periodized programs in different population and age ranges (Rhea, Ball, Phillips, \& Burkett, 2002). One of the most popular periodization models, block periodization (BP), is characterized by an initial high volume and low intensity of training, increasing in intensity and decreasing in volume in the subsequent training phases (Rhea, et al., 2002). BP entails training cycles of highly concentrated workloads, focused on a minimal number of specialized targeted abilities (Issurin, 2010). Another popular type of periodization is 
called, "undulating" or "nonlinear". The most frequently used is daily undulating periodization (DUP), in which modifications of training volume and intensity are made daily (Buford, Rossi, Smith, \& Warren, 2007). DUP includes frequent changes in stimuli and periods of recovery, thereby favoring strength gains (Harries, Lubans, \& Callister, 2015a), although controversial results have been reported by the literature (Hartmann, Bob, Wirth, \& Schmidtbleicher, 2009).

There is growing evidence regarding the importance of integrating strength training in young athletes' programs to improve their health and fitness. Improvements in bone health, motor skills, sports performance, and well-being, together with a decrease in sport-related injuries (Faigenbaum, et al., 2009), are among the advantages reported for young athletes. However, there is limited research concerning the effects of different periodizations on young athletes (Harries, Lubans, \& Callister, 2015b; Moraes, Fleck, Ricardo-Dias, \& Simao, 2013). Despite this, research suggests that children and adolescents are more likely to adhere to a periodized program where there is progression in resistance training than to a non-periodized program (Moraes, et al., 2013). Nevertheless, there is a lack of research comparing BP and DUP programs in young athletes and, to date, only one study has compared both periodization models in this population, concluding that both programs were similarly effective in eliciting maximal strength gains (Harries, et al., 2015b). Of note that Harries et al. (2015b) only evaluated changes in maximal strength and did not measure other performance variables related to sport performance (i.e., jumping ability, sprinting or throwing performance).

The purpose of the present study was to compare two different periodized programs - block periodization (BP) and daily undulating periodization (DUP), in eliciting changes in strength, jumping performance and throwing velocity in young male handball players.

\section{Methods}

\section{Study design}

Two groups of handball players were randomly formed and submitted to either the BP or DUP added to the regular handball training. Total training volume and intensity were equal for both groups. After familiarization with the test procedures, the players performed a physical fitness test battery, before (on two non-consecutive days the week before) (Pre) and after (one week) (Post) eight weeks of training. The test battery consisted of: squat maximal strength (1 repetition maximum [RM]), $20 \mathrm{~m}$ sprint time, triple jump distance with the left and right leg, countermovement jump height (CMJ), $7 \mathrm{~m}$ standing throw, $9 \mathrm{~m}$ jumping throwing velocity and overhead medicine ball throw for distance. To reduce the interference of uncontrolled variables, all the participants were instructed to maintain their habitual lifestyle and normal dietary intake before and during the study. The participants were asked to avoid exercise on the day before a test and to consume their last (caffeine-free) meal at least $2 \mathrm{~h}$ before the scheduled test time.

\section{Participants}

Eighteen young male handball players from two different Spanish clubs and without previous experience in strength training, volunteered to participate in the study. All participants had at least five years of experience in handball training and were competing at regional level. Based on the club they played for, and in order to keep them blinded to the other intervention group, the players were assigned either to the $\mathrm{BP}(\mathrm{n}=9$; age $=15.3 \pm 0.5$ years; body height $=181.2 \pm 5.1 \mathrm{~cm}$; body mass $=73.3 \pm 12.2$ $\mathrm{kg})$ or DUP group $(\mathrm{n}=9$; age $=15.6 \pm 0.5$ years; body height $=179.3 \pm 7.1 \mathrm{~cm}$; body mass $=75.9 \pm 11.9 \mathrm{~kg}$ ). Due to non-compliance with the training and/or testing, only 15 participants were included in the final analysis $(\mathrm{BP}=6$; DUP $=9)$.

Before participating in the study, players were fully informed about the protocol and a written informed consent was obtained from each of them as well as from their parents/guardians. The procedures were approved by the University Miguel Hernandez of Elche Ethics Review Committee conformed to the Code of Ethics of the World Medical Association (Declaration of Helsinki).

Participants did not perform any additional strength training during the study, but continued with the sport-specific team training. This normal training was equal in both groups and consisted of 90 minutes of technical-tactical on-court sessions 3 days/week. As previously mentioned, none of the players in the study had regular experience in strength training and just had some experience (i.e., familiarization sessions) in various forms of plyometric (e.g., medicine ball, hopping) and injuryprevention (e.g., elastic tubing and core training) training.

To ensure standardization of test administration, all the assessments were performed in the same order, using the same testing devices and operators. Training protocols were conducted over an 8-week period beginning at the end of the Christmas break. Test sessions were undertaken between 18:00 and 20:00 h. Before the test procedures a general and specific warm-up (tests realized at submaximal intensity) was performed by the participants.

\section{Maximal dynamic strength}

After three warm-up sets of 5-10 repetitions with an unloaded bar, the participants progressively increased the load across attempts until the $1 \mathrm{RM}$ 
was obtained (Earle \& Baechle, 2004). The rest period between trials was at least five minutes. The squat exercise required the participants to rest the bar on their trapezius and the squat was performed to the parallel position until the trochanter was lower or at the same level as the knee. The participant then lifted the weight until his knees were fully extended.

\section{Jumping performance}

Countermovement vertical jump (CMJ) and a standing triple jump test were used to assess lower body power. The CMJ was performed on a contact platform (Globus, Italy), and height was calculated from the formula $h=g \cdot f t^{2} / 8$ (where $h$ is the jump height in meters; $g$ is the gravity acceleration $\left[9.81 \mathrm{~m} \cdot \mathrm{s}^{-2}\right] ; \mathrm{ft}$ is the flight time in seconds) (Bosco, Luhtanen, \& Komi, 1983). Participants were asked to keep their hands on the hips and a self-selected countermovement depth was chosen by each participant. For the standing triple jump test (Risberg \& Ekeland, 1994), the starting position was support on one leg. Both legs were measured separately (triple right jump [TRJ] and triple left jump [TLJ]). Standing on one leg, the participants jumped three times along a straight line using the same leg. The measured distance was recorded with the heel mark. The participants were allowed to land using one or both legs on the last support. Two attempts were performed of each jump and the best result was used for the analysis.

\section{Throwing performance}

Throwing velocity was measured using a portable radar (Stalker Sport 2, Applied Concepts Inc, USA) with an accuracy of $0.1 \mathrm{~km} \cdot \mathrm{h}^{-1}$. This method has shown a high reliability $(\mathrm{ICC}=0.96 ; \mathrm{CV}=2.4 \%)$ when measuring $9 \mathrm{~m}$ throwing velocity as previously reported by Marques and González-Badillo (2006). After 10 minutes of warm-up, consisting of jogging, dynamic stretches and technical skills (passes and throws at a submaximal velocity), each participant performed two maximal throw attempts using an official handball ball (mass $375 \mathrm{~g}$, circumference $56 \mathrm{~cm}$ ) from both $7 \mathrm{~m}$ (standing throw) and $9 \mathrm{~m}$ line (jumping throw). The standing and jumping throws have been described previously (Hermassi, et al., 2011).

For the overhead medicine ball throw the players stood behind the line with their feet parallel and slightly apart, facing the throwing direction and holding a $5 \mathrm{~kg}$ medicine ball. The ball was brought back behind the head and then thrown vigorously forward as far as possible without the player crossing the line (Stockbrugger \& Haennel, 2001). They were not allowed to step over the line after the throw. Each participant performed two maximal throw attempts and the best one was used for further analysis.

\section{Sprint performance}

Starting position was individually chosen by each participant, a meter behind the photocell gate connected to a digital timer. Each player performed two maximal $20 \mathrm{~m}$ sprints interspersed with three minutes of passive recovery. The best time achieved was retained.

\section{Training protocol}

The strength training program for both groups is described in Table 1. During the training program, the same exercises, volume and intensity were used in both the BP and DUP group. The distribution of the sessions, however, was different between the groups according to their periodization system. The intensity was calculated and individualized based on the initial tests. Every participant was informed about the intensity before each training session, and all the strength sessions were supervised by a qualified member of the research study. In order to be included in the final analysis, participants were required to complete $>80 \%$ of the sessions prescribed.

The DUP group modified their strength training sessions day-to-day. During the first four weeks, the first weekly session focused on hypertrophy and the second one on power strength. In the following two weeks, the weekly schedule was: hypertrophy $\left(1^{\text {st }}\right.$ session) and explosive strength ( $2^{\text {nd }}$ session). Finally, during the last two weeks, the first weekly session focused on power strength and the second on explosive strength.

The BP group followed a periodized program (Buford, 2007), divided into three mesocycles characterized by different volumes and intensities. Thus, the BP program consisted of three weeks of hypertrophy, followed by three weeks of power strength and two weeks of explosive strength.

During every workout three exercises were performed for the upper body, and two for the lower body. A description of the exercises performed in both groups can be seen in Table 2 .

\section{Statistical analysis}

Student's $t$-test was used to detect betweengroup differences in the pre- and post-tests. The normality of data distribution was assessed by the Kolmogorov-Smirnov test. The adaptation to training was analyzed using a general linear model with the repeated-measures ANOVA test, followed by the Bonferroni post hoc test. Statistical analysis were carried out using the statistical package SPSS 18.0 (SPSS Inc, Chicago, IL). Statistical significance was set at $p<.05$, and results were summarized as means \pm SD. Effect sizes (ES) were used to examine the magnitude of the differences at pre- and post-test, and the change over the intervention period. The following criteria were 
Table 1. Training program description for both experimental groups

\begin{tabular}{|c|c|c|c|c|c|}
\hline \multirow[b]{2}{*}{ Week } & \multirow[b]{2}{*}{$\begin{array}{l}\text { Training } \\
\text { session }\end{array}$} & \multicolumn{2}{|c|}{ DUP group } & \multicolumn{2}{|c|}{ BP group } \\
\hline & & Sets $x$ reps $(\% 1 \mathrm{RM})$ & $\begin{array}{l}\text { Resting time } \\
\text { (minutes) }\end{array}$ & Sets $x$ reps $(\% 1 \mathrm{RM})$ & $\begin{array}{l}\text { Resting time } \\
\text { (minutes) }\end{array}$ \\
\hline \multirow{2}{*}{ Week 1} & 1 & $4 \times 10(70 \%)$ & $1-2$ & $4 \times 10(70 \%)$ & $1-2$ \\
\hline & 2 & $4 \times 8(50 \%)$ & $2-3$ & $4 \times 10(70 \%)$ & $1-2$ \\
\hline \multirow{2}{*}{ Week 2} & 3 & $4 \times 10(70 \%)$ & $1-2$ & $4 \times 10(75 \%)$ & $1-2$ \\
\hline & 4 & $4 \times 8(50 \%)$ & $2-3$ & $4 \times 10(75 \%)$ & $1-2$ \\
\hline \multirow{2}{*}{ Week 3} & 5 & $4 \times 10(75 \%)$ & $1-2$ & $5 \times 10(80 \%)$ & $1-2$ \\
\hline & 6 & $4 \times 8(50 \%)$ & $2-3$ & $5 \times 10(80 \%)$ & $1-2$ \\
\hline \multirow{2}{*}{ Week 4} & 7 & $4 \times 10(75 \%)$ & $1-2$ & $4 \times 8(50 \%)$ & $2-3$ \\
\hline & 8 & $4 \times 8(40 \%)$ & $2-3$ & $4 \times 8(50 \%)$ & $2-3$ \\
\hline \multirow{2}{*}{ Week 5} & 9 & $5 \times 10(80 \%)$ & $1-2$ & $4 \times 8(50 \%)$ & $2-3$ \\
\hline & 10 & $6 \times 6(30 \%)$ & 3 & $4 \times 8(50 \%)$ & $2-3$ \\
\hline \multirow{2}{*}{ Week 6} & 11 & $5 \times 10(80 \%)$ & $1-2$ & $4 \times 8(40 \%)$ & $2-3$ \\
\hline & 12 & $6 \times 6(30 \%)$ & 3 & $4 \times 8(40 \%)$ & $2-3$ \\
\hline \multirow{2}{*}{ Week 7} & 13 & $4 \times 8(50 \%)$ & $2-3$ & $6 \times 6(30 \%)$ & 3 \\
\hline & 14 & $6 \times 6(30 \%)$ & 3 & $6 \times 6(30 \%)$ & 3 \\
\hline \multirow{2}{*}{ Week 8} & 15 & $4 \times 8(40 \%)$ & $2-3$ & $6 \times 6(30 \%)$ & 3 \\
\hline & 16 & $6 \times 6(30 \%)$ & 3 & $6 \times 6(30 \%)$ & 3 \\
\hline
\end{tabular}

$1 \mathrm{RM}=$ one repetition maximum

Table 2. Exercises performed in each phase of the periodization program

\begin{tabular}{lll}
\hline Phase & Upper body & Lower body \\
\hline Hypertrophy (4x10 at 80\% 1 RM) & Pull up, bench press, and military press & Squat and lunge \\
Power (4x8 at 50\% 1 RM) & Body row, bench press, and push press & Squat jump and step up \\
Explosive (6x6 at 30\% 1 RM) & $\begin{array}{l}\text { Plyometric body row, plyometric push up, and bench } \\
\text { press throw }\end{array}$ & Lunge jump and step up jump \\
\hline
\end{tabular}

used for the calculated effect sizes: $<0.2=$ trivial, $0.2-0.5=$ small, $>0.5-0.8=$ moderate, and $>0.8=$ large (Rhea \& Alderman, 2004).

\section{Results}

There were no between-group differences at baseline; however, due to dropouts, there was a between-group difference in the $9 \mathrm{~m}$ throw at pretest. Table 3 summarizes the statistical changes in performance following the 8-week period in both the DUP and the BP group.

\section{Maximal dynamic strength}

A significant time effect was detected for squat $1 \mathrm{RM}$. The improvement was significant in both DUP $(+33.3 \% ; p=.001)$ and BP $(+17.4 \% ; p=.007)$. In addition, there was a moderate but non-significant interaction $(\mathrm{ES}=0.91 ; \mathrm{p}=.082)$ between DUP and BP.

\section{Jumping performance}

Significant time effects were observed in the TRJ, in both the DUP $(+4.0 \%, \mathrm{p}=.034)$ and the BP $(+9.7 \%, \mathrm{p}=.017)$ group. In the TLJ, the changes were also significant in both the DUP $(+6.3 \%, \mathrm{p}=.048)$ and the BP group $(+12.7 \%, p=.006)$. Non-significant time $\times$ group interactions $(p>1)$ on this parameter were found.

\section{Throwing performance}

There was a between-groups difference in the 7-meter standing throw $(\mathrm{p}=.041)$ and 9 -meter $(\mathrm{p}=.012)$ jumping throw change from pre- to posttest (time $\times$ group interaction). The $7 \mathrm{~m}$ standing throw velocity was significantly decreased in the DUP group $(-6.8 \% ; \mathrm{ES}=-0.79 ; \mathrm{p}<.01)$, whereas the $\mathrm{BP}$ group did not change. A significant increase in the $9 \mathrm{~m}$ jumping throw velocity was detected in the DUP group $(+10.8 \%$; $\mathrm{p}=.036)$. Changes in this task performance were not significant $(\mathrm{p}=.209)$ in the BP group. For the medicine ball throw, the DUP group significantly improved distance $(+11 \%$, $\mathrm{p}=.015)$, whereas the BP group did not change $(+5.3 \%, \mathrm{p}=.492)$.

\section{Sprinting performance}

No significant changes were found after the training period in the $20 \mathrm{~m}$ sprint time either for the DUP ( $\mathrm{p}=.927)$ or the BP $(\mathrm{p}=.114)$ program. 
Table 3. Changes in performance parameters during the intervention period in both groups

\begin{tabular}{lcccccccc}
\hline & \multicolumn{3}{c}{ DUP } \\
\hline & Pre & Post & $p$ & ES & Pre & Post & p & ES \\
\hline Squat 1 RM $(\mathrm{kg})$ & $60.3 \pm 9.1$ & $80.4 \pm 9.1^{* *}$ & .001 & 1.48 & $63.3 \pm 6.5$ & $74.3 \pm 4.6^{* *}$ & .007 & 1.40 \\
CMJ $(\mathrm{cm})$ & $32.7 \pm 5.8$ & $32.5 \pm 4.3$ & .930 & -0.04 & $31.4 \pm 5.0$ & $32.9 \pm 3.0$ & .543 & 0.38 \\
TRJ $(\mathrm{m})$ & $6.1 \pm 0.9$ & $6.4 \pm 0.7^{*}$ & .044 & 0.32 & $5.9 \pm 0.3$ & $6.4 \pm 0.3^{* *}$ & .017 & 1.40 \\
TLJ $(\mathrm{m})$ & $6.2 \pm 0.8$ & $6.4 \pm 0.7^{*}$ & .048 & 0.51 & $5.8 \pm 0.3$ & $6.5 \pm 0.4^{* *}$ & .006 & 1.40 \\
7m throw $\left(\mathrm{km} \cdot \mathrm{h}^{-1}\right)$ & $78.4 \pm 7.6$ & $73.1 \pm 4.7^{* *} \dagger$ & .009 & -0.79 & $74.8 \pm 1.6$ & $74.3 \pm 3.7$ & .767 & -0.18 \\
9m throw $\left(\mathrm{km} \cdot \mathrm{h}^{-1}\right)$ & $68.2 \pm 7.3^{\#}$ & $75.6 \pm 6.4^{*} \dagger$ & .036 & 0.96 & $79.0 \pm 8.6$ & $73.8 \pm 4.0$ & .209 & -0.75 \\
Med ball $(\mathrm{m})$ & $5.8 \pm 0.8$ & $6.4 \pm 0.9^{*}$ & .015 & 0.74 & $5.7 \pm 0.5$ & $6.0 \pm 0.9$ & .492 & 0.37 \\
20m sprint $(\mathrm{s})$ & $3.2 \pm 0.2$ & $3.2 \pm 0.2$ & .927 & 0.11 & $3.3 \pm 0.1$ & $3.2 \pm 0.1$ & .114 & -0.51 \\
\hline
\end{tabular}

Note. TRJ = triple right-leg jump; TLJ = triple left-leg jump; Med. = medicine; $\#=$ between-group difference at pre-test; $\dagger=$ betweengroup difference in pre-post $(p<.05) ;{ }^{*}$ within-group difference $\left({ }^{*}=p<.05 ;{ }^{* *}=p<.01\right)$.

\section{Discussion and conclusions}

To the best of our knowledge, this is the first study to compare two periodization models (i.e., BP and DUP) in young male handball players. The major finding of the present study was that both periodization groups significantly increased their maximal strength, as tested by the 1 RM squat, and their jumping performance in the triple jump exercise to a similar extent.

In the last decade, several studies confirmed the importance of integrating strength training into the development of young athletes (Faigenbaum, et al., 2009; Faigenbaum, Lloyd, McDonald, \& Myer, 2016; Harries, Lubans, \& Callister, 2012; Lloyd, Oliver, Faigenbaum, Myer, \& De Ste Croix, 2014). Moraes et al. (2013) showed that a periodized program using a daily undulating model elicited at least similar gains, compared to a non-periodized program. Later on, Harries et al. (2015a) compared the adaptations to different periodized programs in adolescent rugby players, showing that both periodized programs (BP and DUP) were similarly effective in eliciting maximal strength gains. Our results are in line with these studies, as both the BP and the DUP significantly increased the squat maximal strength, but to a different extent $(\mathrm{BP}=17.4 \%$; $\mathrm{DUP}=33.3 \%)$, possibly suggesting that a more frequent variation in the weekly strength training (DUP) may elicit superior gains ( $E S=.91$ ), although it did not reach statistical significance in the current study ( $\mathrm{p}=.082$ ). These results are important for handball players because higher values of maximal strength and explosive strength would give a clear advantage in performance of handball-specific movements. Thus, significant positive correlations between power production in the halfsquat exercise and throwing velocity in the threestep running throw have been reported (Gorostiaga, et al., 2005).

Jumping performance is a classical test to assess lower limb explosive strength (Bosco, et al., 1983).
Thus, improvements in this ability may lead to improvements in on-court performance (Hermassi, et al., 2011). In the present study, CMJ did not show significant improvements after the training intervention. This is consistent with other studies involving adolescents (Moraes, et al., 2013) or elite handball players (Gorostiaga, Izquierdo, Iturralde, Ruesta, \& Ibañez, 1999). Maybe, the effects of strength gains after a resistance training intervention during the handball season could be observed in a more sportspecific (and commonly used) jumping task such as a jump throw including a run-up (Michalsik, Aagaard, \& Madsen, 2011).

On the other hand, triple jump distance, using both the left and right leg, significantly improved in the BP $(\mathrm{ES}=1.40)$ and the DUP group ( $\mathrm{ES}=0.32$ 0.51 ). The improvement on this variable, although non-significantly different between groups, was slightly greater in the BP. Some authors have suggested that horizontal jumping ability seems an important variable for evaluating young handball players (Lidor, et al., 2005; Maulder \& Cronin, 2005). Thus, both programs seemed to be effective in improving this key variable for handball performance. No significant differences between improvements caused by the two periodization models may indicate that in the sample of the current study (adolescent players), training variables such as total volume and intensity (equal in both groups in this study) could be more influential for performance improvements, with the periodization model playing a minor role.

As regards throwing performance, the DUP group significantly improved their performance in the $9 \mathrm{~m}$ jump throw and in the medicine ball throw, while there was a significant decrease in the $7 \mathrm{~m}$ standing throw. In the BP group there were no changes in any throwing test. The reason for the greater improvement in the DUP group in both the $9 \mathrm{~m}$ and the medicine ball throw may be related to frequent changes in stimuli (different aim in each session) that are advantageous in stressing 
the nervous system and increasing body strength (Fleck, 2011; Harries, et al., 2015b). It could be hypothesized that training with variations could be more useful for upper limbs, because the interaction with the on-court training is lower than for lower limb. In addition, daily changes in DUP could divert monotony for athletes, leading to their higher motivation during training sessions, and may play a potential role in performance improvements after a training period. On the other hand, the $7 \mathrm{~m}$ standing throw may not be affected by the strength programs proposed for the BP and DUP groups.

Although sprinting speed has been reported as an important variable for the performance of teamhandball players (Zapartidis, Vareltzis, Gouvali, \& Kororos, 2009), the sprint test did not show significant changes in either group after the intervention. Team handball, however, is characterized by frequent short accelerations, decelerations, and changes of direction (Povoas, et al., 2012). The effects of a periodized strength training program may be more evident in tests involving more suitable tasks for measuring team-handball performance (Lidor, et al., 2005; Wagner, Finkenzeller, Würth, \& von Duvillard, 2014). Therefore, future studies should evaluate adaptations using more specific assessments, including decelerations and changes of directions.

Both the BP and DUP periodizations significantly improved maximal strength and jumping ability in adolescent handball players. Nevertheless, the DUP periodization seems to be a superior stimulus for improving explosive force in upper limbs, perhaps due to minor interactions with court situations. Coaches interested in optimizing strength training for young handball players should be aware that resistance training programs following a daily undulating periodization model could lead to superior gains in throwing performance compared to block periodized programs.

\section{References}

Bosco, C., Luhtanen, P., \& Komi P.V. (1983). A simple method for measurement of mechanical power in jumping. European Journal of Applied Physiology, 50(2), 273-282.

Buford, T.W., Rossi, S.J., Smith, D.B, \& Warren, A.J. (2007). A comparison of periodization models during nine weeks with equated volume and intensity for strength. Journal of Strength and Conditioning Research, 21(4), 1245-1250.

Chelly, M.S., Hermassi, S., \& Shephard, R.J. (2010). Relationships between power and strength of the upper and lower limb muscles and throwing velocity in male handball players. Journal of Strength and Conditioning Research, 24(6), 1480-1487.

Earle, R.W., \& Baechle, T.R. (2004). NSCA's essentials of personal training. Champaign, IL: Human Kinetics.

Faigenbaum, A.D., Kraemer, W.J., Blimkie, C.J., Jeffreys, I., Micheli, L.J., Nitka, M., \& Rowland, T.W. (2009). Youth resistance training: Updated position statement paper from the National Strength and Conditioning Association. Journal of Strength and Conditioning Research, 23(5), 60-79.

Faigenbaum, A.D., Lloyd, R.S., MacDonald, J., \& Myer, G.D. (2016). Citius, altius, fortius: Beneficial effects of resistance training for young athletes. British Journal of Sports Medicine, 50(1), 3-7.

Fleck, S.J. (1999). Periodized strength training: A critical review. Journal of Strength and Conditioning Research, 13(1), $82-89$.

Fleck, S.J. (2011). Non-linear periodization for general fitness and athletes. Journal of Human Kinetics, 29 , 41-45.

Gorostiaga, E.M., Granados, C., Ibañez, J., Gonzalez-Badillo, J.J., \& Izquierdo, M. (2006). Effects of an entire season on physical fitness changes in elite male handball players. Medicine and Science in Sports and Exercise, 38(2), 357-366.

Gorostiaga, E.M., Granados, C., Ibañez, J., \& Izquierdo, M. (2005). Differences in physical fitness and throwing velocity among elite and amateur male handball players. International Journal of Sports Medicine, 26(3), 225-232.

Gorostiaga, E.M., Izquierdo, M., Iturralde, P., Ruesta, M., \& Ibáñez, J. (1999). Effects of heavy resistance training on maximal and explosive force production, endurance and serum hormones in adolescent handball players. European Journal of Applied Physiology, 80(5), 485-493.

Granados, C., Izquierdo, M., Ibañez, J., Ruesta, M., \& Gorostiaga, E. (2008). Effects of an entire season on physical fitness in elite female handball players. Medicine and Science in Sports and Exercise, 40(2), 351-361.

Harries, S.K., Lubans, D.R., \& Callister, R. (2012). Resistance training to improve power and sports performance in adolescent athletes: A systematic review and meta-analysis. Journal of Science and Medicine in Sport, 15(6), 532-540.

Harries, S.K., Lubans, D.R., \& Callister, R. (2015a). Systematic review and meta-analysis of linear and undulating periodized resistance training programs on muscular strength. Journal of Strength and Conditioning Research, 29(4), 1113-1125.

Harries, S.K., Lubans, D.R., \& Callister, R. (2015b). Comparison of resistance training progression models on maximal strength in sub-elite adolescent rugby union players. Journal of Science and Medicine in Sport, 19(2), 163-169. 
Hartmann, H., Bob, A., Wirth K., \& Schmidtbleicher, D. (2009). Effects of different periodization models on rate of force development and power ability of the upper extremity. Journal of Strength and Conditioning Research, 23(7), 1921-1932.

Hermassi, S., Chelly, M.S., Tabka, Z., Shephard, R.J., \& Chamari, K. (2011). Effects of 8-week in-season upper and lower limb heavy resistance training on the peak power, throwing velocity, and sprint performance of elite male handball players. Journal of Strength and Conditioning Research, 25(9), 2424-2433.

Issurin, V.B. (2010). New horizons for the methodology and physiology of training periodization. Sports Medicine, 40(3), 189-206.

Lidor, R., Falk, B., Arnon, M., Cohen, Y., Segal, G., \& Lander, Y. (2005). Measurement of talent in team handball: The questionable use of motor and physical tests. Journal of Strength and Conditioning Research, 19(2), 318-325.

Lloyd, R.S., Oliver, J.L., Faigenbaum, A.D., Myer, G.D., \& De SteCroix, M.B. (2014). Chronological age vs. biological maturation: Implications for exercise programming in youth. Journal of Strength and Conditioning Research, 28(5), 1454-1464.

Marques, M.C, \& González-Badillo, J.J. (2006). In-season resistance training and detraining in professional team handball players. Journal of Strength and Conditioning Research, 20(3), 563-571.

Marques, M.C., Van Den Tillaar, R., Vescovi, J.D., \& González-Badillo, J.J. (2007). Relationship between throwing velocity, muscle power, and bar velocity during bench press in elite handball players. International Journal of Sports Physiology and Performance, 2(4), 414-422.

Maulder, P., \& Cronin, J. (2005). Horizontal and vertical jump assessment: Reliability, symmetry, discriminative and predictive ability. Physical Therapy in Sport, 6(2),74-82.

Michalsik, L.B., Aagaard, P., \& Madsen, K. (2011). Technical activity profile and influence of body anthropometry in male elite team handball players. Journal of Strength and Conditioning Research, 29(4), 1126-1138.

Moraes, E., Fleck, S.J., Ricardo-Dias, M., \& Simao, R. (2013). Effects on strength, power, and flexibility in adolescents of nonperiodized vs. daily nonlinear periodized weight training. Journal of Strength and Conditioning Research, 27(12), 3310-3321.

Povoas, S.C., Seabra, A.F., Ascensao, A., Magalhaes, J., Soares, J.M., \& Rebelo, A.N.(2012). Physical and physiological demands of elite team handball. Journal of Strength and Conditioning Research, 26(12), 3365-3375.

Rhea, M.R., \& Alderman, B.L. (2004). A meta-analysis of periodized versus nonperiodized strength and power training programs. Research Quarterly for Exercise and Sport, 75(4), 413-422.

Rhea, M.R., Ball, S.D., Phillips, W.T., \& Burkett, L.N. (2002). A comparison of linear and daily undulating periodized programs with equated volume and intensity for strength. Journal of Strength and Conditioning Research, 16(2), 250-255.

Risberg, M.A., \& Ekeland, A. (1994). Assessment of functional tests after anterior cruciate ligament surgery. Journal of Orthopaedic Sports and Physical Therapy, 19(4), 212-217.

Stockbrugger, B.A., \& Haennel, R.G. (2001). Validity and reliability of a medicine ball explosive power test. Journal of Strength and Conditioning Research, 15(4), 431-438.

Wagner, H., Finkenzeller, T., Würth, S., \& von Duvillard, S.P. (2014). Individual and team performance in teamhandball: A review. Journal of Sports Science and Medicine, 13(4), 808-816.

Zapartidis, I., Vareltzis, I., Gouvali, M., \& Kororos, P. (2009). Physical fitness and anthropometric characteristics in different levels of young handball players. Open Sports Science Journal, 2(1), 22-28.

Submitted: November 23, 2016

Accepted: September 8, 2017

Published Online First: January 16, 2018

Correspondence to:

Jose Luis Hernández Davó, Ph.D.

Centro de Investigación del Deporte, Universidad

Miguel Hernández de Elche

Avda. de la Universidad s/n. 03202. Elche (Alicante),

Spain

Phone: +34662048064

E-mail: jlhdez43@gmail.com

\section{Disclosure statement}

No potential conflict of interest was reported by the authors.

\section{Funding}

No funding was received to carry out this study. 